\title{
A Fortran program for calculating electron or hole mobility in disordered semiconductors from first-principles
}

\author{
Zi Li, Xu Zhang, Gang Lu* \\ Department of Physics and Astronomy, California State University Northridge, Northridge, CA 91330-8268, USA
}

\section{A R T I C L E I N F O}

\section{Article history:}

Received 6 April 2011

Accepted 13 July 2011

Available online 26 July 2011

\section{Keywords:}

Carrier mobility

Disordered semiconductors

First-principles

\begin{abstract}
A B S T R A C T
A Fortran program is developed to calculate charge carrier (electron or hole) mobility in disordered semiconductors from first-principles. The method is based on non-adiabatic ab initio molecular dynamics and static master equation, treating dynamic and static disorder on the same footing. We have applied the method to calculate the hole mobility in disordered poly(3-hexylthiophene) conjugated polymers as a function of temperature and electric field and obtained excellent agreements with experimental results. The program could be used to explore structure-mobility relation in disordered semiconducting polymers/organic semiconductors and aid rational design of these materials.
\end{abstract}

\section{Program summary}

Program title: FPMu

Catalogue identifier: AEJV_v1_0

Program summary URL: http://cpc.cs.qub.ac.uk/summaries/AEJV_v1_0.html

Program obtainable from: CPC Program Library, Queen's University, Belfast, N. Ireland

Licensing provisions: Standard CPC licence, http://cpc.cs.qub.ac.uk/licence/licence.html

No. of lines in distributed program, including test data, etc.: 788580

No. of bytes in distributed program, including test data, etc.: 8433024

Distribution format: tar.gz

Programming language: Fortran 90

Computer: Any architecture with a Fortran 90 compiler

Operating system: Linux, Windows

RAM: Proportional to the system size, in our example, $1.2 \mathrm{~GB}$

Classification: 7.9

Nature of problem: Determine carrier mobility from first-principles in disordered semiconductors as a function of temperature, electric field and carrier concentration.

Solution method: Iteratively solve master equation with carrier state energy and transition rates determined from first-principles.

Restrictions: Mobility for disordered semiconductors where the carrier wave-functions are localized and the carrier transport is due to phonon-assisted hopping mechanism.

Running time: Depending on the system size (about an hour for the example here).

(C) 2011 Elsevier B.V. All rights reserved.

\section{Introduction}

Disordered semiconducting polymers and organic semiconductors have recently received significant attention for their potential applications in light-emitting diodes [1], field-effect transistors [2],

\footnotetext{
th This paper and its associated computer program are available via the Computer Physics Communications homepage on ScienceDirect (http://www.sciencedirect. com/science/journal/00104655)

* Corresponding author.

E-mail address: ganglu@csun.edu (G. Lu).
}

and photovoltaics [3], etc. However, one of the major bottlenecks that limit the efficiency of the disordered semiconductor devices is their poor charge carrier mobility [4]. Therefore it is of great scientific and technological importance to understand the charge transport process and in particular to develop theoretical tools that can predict the carrier mobility from first-principles. Here, we present a Fortran program that can determine charge carrier (electron or hole) mobility in disordered conjugated polymers and organic semiconductors as a function of temperature, electric field and carrier concentration, entirely from first-principles; i.e., there is no empirical input or adjustable parameter in the simulations. In this 
program, ab initio non-adiabatic molecular dynamics (NAMD) [5] is performed to simulate phonon-assisted electron transitions between localized electronic states in the disordered system and the electronic energy levels are determined from first-principles BornOppenheimer molecular dynamics (BOMD) on-the-fly. In conjunction with a macroscopic master equation [6], this program can predict the carrier mobility of disordered semiconductors in which the carrier wave-functions are localized and the charge transfer is assisted by phonons. Here, we determine the hole mobility in amorphous poly(3-hexylthiophene) (P3HT) polymer as an example.

\section{Theoretical background}

In this section, we briefly summarize the theoretical background of the program as its technical detail has been published elsewhere [7]. The entire system of interest is divided into $N_{g x} \times N_{g y} \times N_{g z}$ cubes, and each cube (or site) should be chosen as large as computationally feasible, but the minimal dimensions of the cube should be greater than the inter-site distance used in the well-known Gaussian mobility models [8]. One of these cubes is designated as the home cube for which the BOMD is performed based on the density functional theory (DFT). The Kohn-Sham (KS) energy levels and wave-functions of the carrier are calculated at each time-step of BOMD and are subsequently used to determine the inter-state transition rates. To simulate the structural disorder, the energy levels and the wave-functions at other cubes are obtained by selecting the wave-functions and the corresponding energies from those at the home cube in a random snapshot (or time-step), and the wave-functions are then randomly rotated before placed into the respective cube [7]. In this way, the KS energy levels and orbitals in the entire disordered system can be approximately constructed by performing the BOMD calculations for the home cube only.

\subsection{The inter-state transition rate}

The inter-state or intra-cube transition rates are obtained from the expansion coefficients of the time-dependent carrier wavefunctions based on the NAMD [7]. The time-dependent oneelectron wave-function of the carrier (electron or hole), $\psi_{i}(\mathbf{r}, t)$, is expanded in terms of adiabatic KS orbitals $\phi_{i}(\mathbf{r}, \mathbf{R}(t))$ :

$\psi_{i}(\mathbf{r}, t)=\sum_{j} c_{j}^{(i)}(t) \phi_{j}(\mathbf{r}, \mathbf{R}(t))$

where $c_{j}^{(i)}(t)$ is the expansion coefficient; $\phi_{i}(\mathbf{r}, \mathbf{R}(t))$ is the eigenstate of the KS Hamiltonian at the instantaneous ionic positions $R(t)$. The label $i$ indicates that at $t=0, \psi_{i}(\mathbf{r}, 0)=\phi_{i}(\mathbf{r}, \mathbf{R}(0))$. The sum is over $N_{c}$ electronic states that are chosen for a given system. The coefficient $c_{j}^{(i)}(t)$ is determined by a second-order finitedifference scheme of the time-dependent KS equation [5]:

$c_{j}^{(i)}\left(t+\Delta t^{\prime}\right)=c_{j}^{(i)}\left(t-\Delta t^{\prime}\right)-2 \Delta t^{\prime} \sum_{k} c_{k}^{(i)}(t)\left(\frac{i}{\hbar} \epsilon_{k} \delta_{j k}+d_{j k}\right)$,

where $\epsilon_{k}$ is the energy of the $k$ th KS orbital; $\Delta t^{\prime}$ is the electronic time-step, typically $10^{-3}$ fs. $d_{j k}$ is the non-adiabatic (NA) coupling between the $j$ th and $k$ th KS orbitals:

$d_{j k} \approx \frac{1}{2 \Delta t}\left(\left\langle\phi_{j}(t) \mid \phi_{k}(t+\Delta t)\right\rangle-\left\langle\phi_{j}(t+\Delta t) \mid \phi_{k}(t)\right\rangle\right)$,

where $\Delta t$ is the ionic time-step, typically $1 \mathrm{fs}$. The transition rate from the state $i$ to $j$ is thus given by $\left\langle\left|c_{j}^{(i)}(t)\right|^{2} / t\right\rangle$, averaging over one NAMD trajectory, and can be calculated as

$$
\begin{aligned}
\gamma_{i, j}^{0} & =\left\langle\left|c_{j}^{(i)}(t)\right|^{2} / t\right\rangle \\
& \simeq \frac{1}{N_{p}} \sum_{k=1}^{N_{p}} \frac{\left|c_{j}^{(i)}\left(k N_{0} \Delta t / N_{p}\right)\right|^{2}}{k N_{0} \Delta t / N_{p}},
\end{aligned}
$$

where $N_{p}$ is the number of sampling points in $N_{0} \Delta t$ NAMD trajectory.

\subsection{The position of KS orbitals}

To assist the evaluation of the transition rates across neighboring cubes, we associate each localized state with a position vector in the real space. First, we calculate the average position of the localized state $i$ by the first moment of its charge density via

$\overline{\mathbf{r}_{i}}=\int \rho_{i}(\mathbf{r}) \mathbf{r} d \mathbf{r}$,

where $\rho_{i}(\mathbf{r})=\left|\phi_{i}(\mathbf{r})\right|^{2}$ is the charge density of the state $i$. Secondly, we determine the root mean square deviation of the average position $\delta \mathbf{r}_{i}$ by

$\delta \mathbf{r}_{i}^{2}=\int \rho_{i}(\mathbf{r})(\mathbf{r}-\overline{\mathbf{r}})^{2} d \mathbf{r}=\overline{\mathbf{r}_{i}^{2}}-\overline{\mathbf{r}}_{i}^{2}$,

with

$\overline{\mathbf{r}_{i}^{2}}=\int \rho_{i}(\mathbf{r}) \mathbf{r}^{2} d \mathbf{r}$.

Finally, the spatial position of the localized state can be obtained by a random selection from the range $\left[\overline{\mathbf{r}_{i}}-\delta \mathbf{r}_{i}, \overline{\mathbf{r}_{i}}+\delta \mathbf{r}_{i}\right]$.

\subsection{The inter-site transition rate}

The inter-site or inter-cube transition rate $\gamma_{i, j^{\prime}}^{0}$, from the state $i$ in cube $n$ to the state $j^{\prime}$ in cube $m$, is determined from the relevant intra-cube transition rates as

$\gamma_{i, j^{\prime}}^{0}=\left(\gamma_{i, j}^{0}+\gamma_{i^{\prime}, j^{\prime}}^{0}\right) / 2$,

where the states $i^{\prime}$ and $j$ are selected from the cubes $n$ and $m$ respectively so that the distances $R_{i j}$ and $R_{i^{\prime} j^{\prime}}$ match as closely as possible to $R_{i j^{\prime}}$. Since the states $i$ and $j\left(i^{\prime}\right.$ and $j^{\prime}$ ) fall within the same cube $n(m)$, their intra-cube transition rate is calculated by Eq. (4).

To ensure the detailed balance, the thermal equilibrium microscopic transition rate $\gamma_{i, j^{\prime}}$ is defined as [9]

$\gamma_{i, j^{\prime}}= \begin{cases}\gamma_{i, j^{\prime}}^{0} \exp \left(-\frac{\epsilon_{j^{\prime}}-\epsilon_{i}}{k_{B} T}\right), & \text { if } \epsilon_{j^{\prime}} \geqslant \epsilon_{i}, \\ \gamma_{i, j^{\prime}}^{0}, & \text { if } \epsilon_{j^{\prime}}<\epsilon_{i} .\end{cases}$

Here $\epsilon_{i}$ and $\epsilon_{j^{\prime}}$ are the eigenvalues of the states $i$ and $j^{\prime}$ in the cubes $n$ and $m$, respectively. The energy difference $\epsilon_{j^{\prime}}-\epsilon_{i}$ in $\gamma_{i, j^{\prime}}$ contains $-e E R_{n, m}^{X}$ and a uniform electric field $E$ is applied in $x$ direction; $R_{n, m}$ is the distance between the cubes $n$ and $m$.

The macroscopic transition rate of the charge carrier from the cube $n$ to the cube $m, \Gamma_{n \rightarrow m}$ is given by summing up the microscopic transition rates:

$\Gamma_{n \rightarrow m}=\sum_{i \in n} p_{n} f_{i} \sum_{j^{\prime} \in m} \gamma_{i, j^{\prime}}\left(1-p_{m} f_{j^{\prime}}\right)$,

where the summations of $i$ and $j^{\prime}$ are over $N_{c}$ electronic states of the carrier in the cubes $n$ and $m$, respectively; $p_{n}$ is the partial concentration or density of the carriers in the cube $n$, and $f_{i}$ is the Fermi-Dirac occupation of the state $i$. Note that the carrier concentration $p_{n}$ is unknown and needs to be determined selfconsistently by solving a master equation, as described next. 


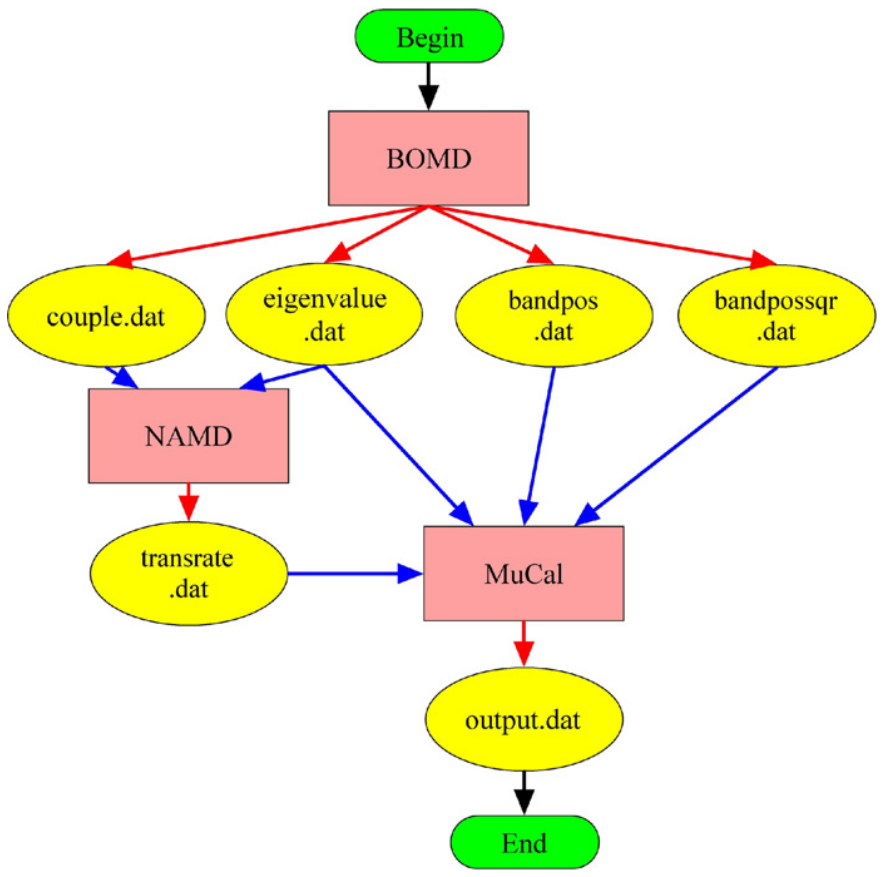

Fig. 1. The flowchart of the program.

\subsection{Master equation for mobility calculation}

In equilibrium, the static master equation

$\sum_{m}\left[\Gamma_{n \rightarrow m}-\Gamma_{m \rightarrow n}\right]=0$,

is satisfied for each cube $n$. The partial concentration $p_{n}$ can be determined from the master equation under the constraint that $\sum_{n} p_{n}=p V$, where $p$ is the total carrier concentration (or density) and $V$ is the volume of the entire system. Therefore the carrier mobility $\mu$ is given by

$\mu=\frac{\sum_{n, m} \Gamma_{n \rightarrow m} R_{n, m}^{x}}{p E V}$.

Numerically, the master equation is solved iteratively with an initial guess of $p_{n}$. By defining

$X_{n \rightarrow m}=\sum_{i} f_{n i} \sum_{j} \gamma_{n i, m j}, \quad$ and

$Y_{n \rightarrow m}=\sum_{i} f_{n i} \sum_{j} \gamma_{n i, m j} f_{m j}$

the macroscopic transition rate from the cube $n$ to the cube $m$ can be rewritten as

$\Gamma_{n \rightarrow m}=p_{n}\left(X_{n \rightarrow m}-p_{m} Y_{n \rightarrow m}\right)$.

Substituting it to Eq. (11), we can determine the partial concentration $p_{n}$ by

$p_{n}=\frac{\sum_{m} X_{m \rightarrow n}}{\sum_{m} X_{n \rightarrow m}-p_{m} \sum_{m}\left(Y_{n \rightarrow m}-Y_{m \rightarrow n}\right)}$.

\section{Introduction to the program}

The program consists of three main codes. The first code is called BOMD, which is a standard DFT-based Born-Oppenheimer molecular dynamics (MD). The BOMD code is not available for download because it is based on a proprietary software VASP (Vienna Ab-initio Simulation Package) [10]. However, there are open

source software such as ABINIT [11], QUANTUM-ESPRESSO [12], SIESTA [13], CPMD [14] and PEtot [15], etc., which can perform the same function as the BOMD code. The relevant information on the KS energy levels and orbitals are extracted from the BOMD snapshots and the inter-state transition rates are determined by the time-dependent non-adiabatic molecular dynamics code, called TDNA code. Finally, the mobility is calculated by solving the master equation with the MuCal code. The flowchart of the entire program is shown in Fig. 1.

\subsection{Ab initio Born-Oppenheimer molecular dynamics}

\subsubsection{Extract information from BOMD code}

In this code, the standard DFT BOMD simulation for the home cube is performed. The KS eigenvalues, NA coupling matrix elements and the KS orbital positions are obtained for each BOMD snapshot. Both the NA coupling matrix elements and the KS orbital positions are determined from the relevant KS orbitals via Eqs. (3), (5) and (7). Note that for the NA coupling, the wave-functions of two adjacent BOMD snapshots or time-steps are needed. For these quantities, only the top $N_{c}$ valance bands (or the bottom $N_{c}$ conduction bands) are considered for the hole (or electron) mobility.

\subsubsection{BOMD output}

The output from the BOMD code consists of four files: couple.dat, eigenvalue.dat, bandpos.dat and bandpossqr.dat. Their format is listed below:

eigenvalue.dat. The eigenvalues of the relevant KS states in each BOMD time-step. There is one comment line with the string "new step" followed by $N_{c}$ eigenvalues (real variables) for each BOMD time-step:

$$
\left.\begin{array}{c}
\text { new step } \\
\epsilon_{1} \\
\epsilon_{2} \\
\vdots \\
\epsilon_{N_{c}}
\end{array}\right\} N_{c}
$$

The subscripts 1 to $N_{c}$ represent $N_{c}$ KS states, and the order of these states should be the same in all the other files.

bandpos.dat. The Cartesian coordinates of the KS states for each BOMD time-step calculated from Eq. (5). There is one comment line with the string "new step" followed by $N_{c}$ lines. In each line, the $x, y$ and $z$ coordinates (real variables) of a KS state are given for each time-step, as shown below:

$\left.\begin{array}{ccc}\text { new step } & \\ x_{1} & y_{1} & z_{1} \\ x_{2} & y_{2} & z_{2} \\ \vdots & & \\ x_{N_{c}} & y_{N_{c}} & z_{N_{c}}\end{array}\right\} N_{c}$

new step

bandpossqr.dat. The square of the KS state coordinates (real variables) for each BOMD time-step calculated from Eq. (7). The format of this file is the same as bandpos.dat.

couple.dat. The NA coupling matrix for each BOMD time-step. There is one comment line with the string "new step" followed by $N_{c} \times N_{c}$ matrix elements (complex variables) with one in each line, as shown below: 


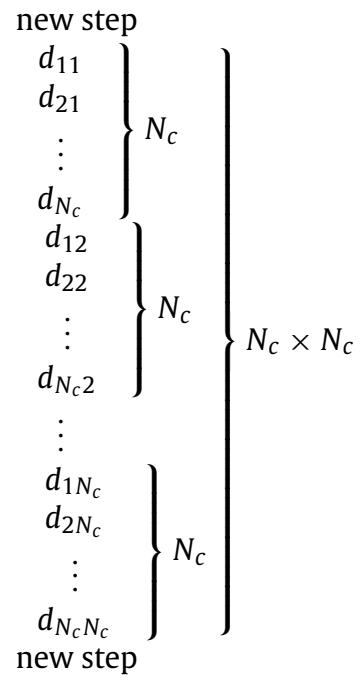

\subsection{TDNA code}

\subsubsection{The structure of TDNA code}

In the TDNA code, the NAMD simulation of the selected states is carried out, and the expansion coefficients are obtained to determine the inter-state transition rates. To use this code, compile the files in the folder $\backslash \operatorname{src} \backslash T D N A$ and then run the executable file TDNA.exe.

The code contains four files: paras.f90 specifies the input parameters; iofiles.f90 reads input and produces output files; for_coefficient.f90 contains the subroutines to perform the time evolution of the electronic states of the carrier; and main.f90 calls subroutines to perform the calculation. The flowchart of main.f90 is shown in Fig. 2, and some important subroutines in for_coefficient.f90 are listed below.

Subroutine evolve_coefficient: evolves the expansion coefficients in $\Delta t$ according to Eq. (2) with the time-step $\Delta t^{\prime}$.

Subroutine cal_transition_rate: calculates the transition rates based on Eq. (4) with the expansion coefficients.

\subsubsection{TDNA input}

The input files include eigenvalue.dat and couple.dat which are obtained from the BOMD code, and parameter.dat which specifies the parameters in the code. A typical parameter.dat is listed below, followed by the meaning of the parameters. The variable names in the code are shown behind the parameters:

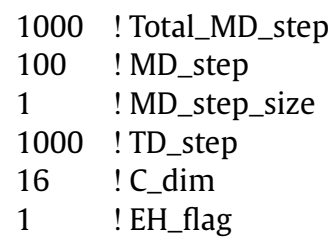

Total_MD_step. The total number of time-steps in the BOMD simulation.

MD_step. The number of time-steps in each NAMD trajectory; it corresponds to $N_{0}$ in Eq. (4).

MD_step_size. The time-step in the BOMD simulation; it corresponds to $\Delta t$ in Eq. (3).

TD_step. The number of NAMD time-steps in one BOMD timestep, i.e., $\Delta t / \Delta t^{\prime}$

C_dim. The number of KS orbitals in the expansion of the timedependent wave-functions of the carrier $\left(N_{C}\right)$.

EH_flag. The flag indicates whether the simulation is for electron or hole. 1 is for hole, and other integers are for electron.

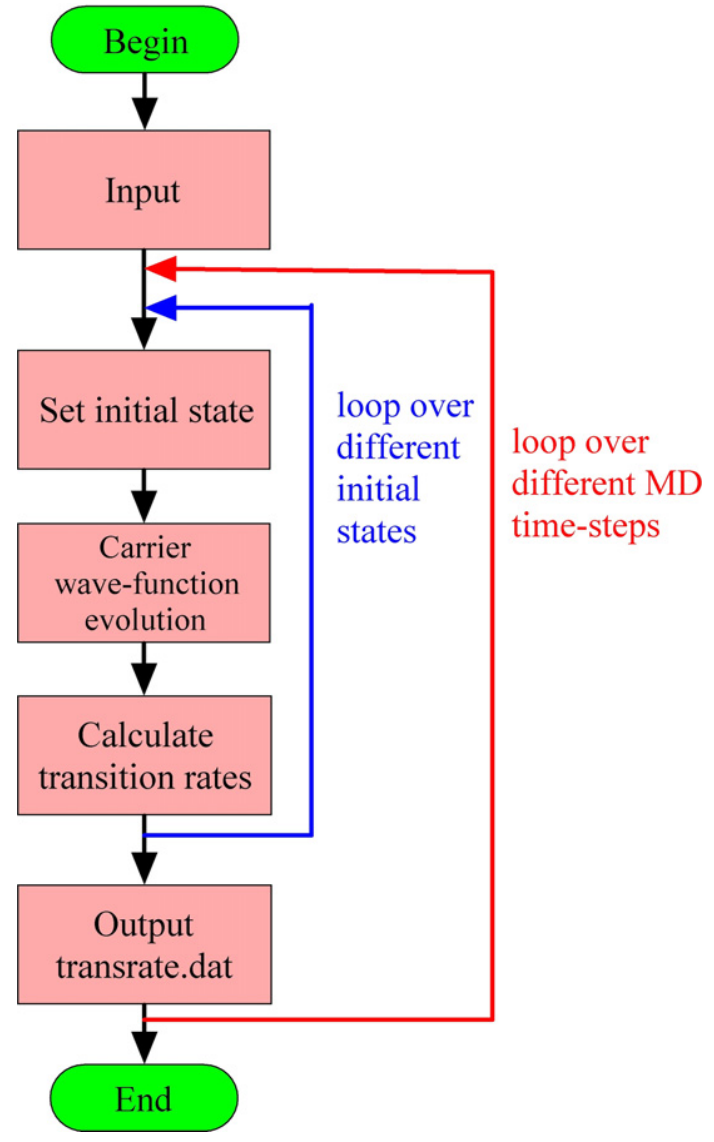

Fig. 2. The flowchart of the TDNA code.

\subsubsection{TDNA output}

The output of the TDNA code is transrate.dat. In this file, the calculated transition rates from one state to another according to Eq. (4) are listed. The rate from one state to itself is set to be zero. For each MD time-step, there is one comment line with the string "new step" followed by $N_{c} \times N_{c}$ transition rates (real variables) with one in each line. The file format is the same as in couple.dat but the entries are real numbers.

\subsection{Carrier mobility calculation by the master equation}

\subsubsection{The structure of MuCal code}

In the MUCal code, the master equation is solved iteratively to calculate the carrier mobility of the macroscopic system. To use the code, compile the files in the folder $\backslash$ src $\backslash M u C a l$ and then run the executable file MuCal.exe.

The code contains four files: parameters.f90 specifies the parameters used in the calculation; random.f90 generates a random number from $(0,1)$; mobility.f90 contains the subroutines to construct the disordered macroscopic system, solve the master equation and calculate the carrier mobility; and main.f90 calls subroutines to perform the mobility calculation. The flowchart of main.f90 is shown in Fig. 3, and some important subroutines in mobility.f90 are listed below:

Subroutine gener_nb: generates the neighbor list of each cube.

Subroutine init grnode: reads energies, positions and transition rates of each BOMD time-step, and assigns them randomly to every cube.

Subroutine calc_Wij: calculates $X_{n \rightarrow m}$ and $Y_{n \rightarrow m}$ in Eqs. (13) and (14).

Subroutine calc_Pi: solves the master equation iteratively and obtains the partial carrier concentration in each cube. 


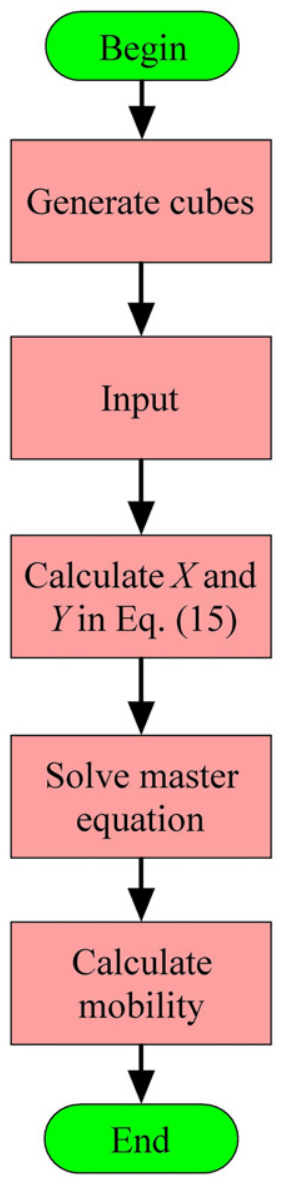

Fig. 3. The flowchart of the MuCal code.
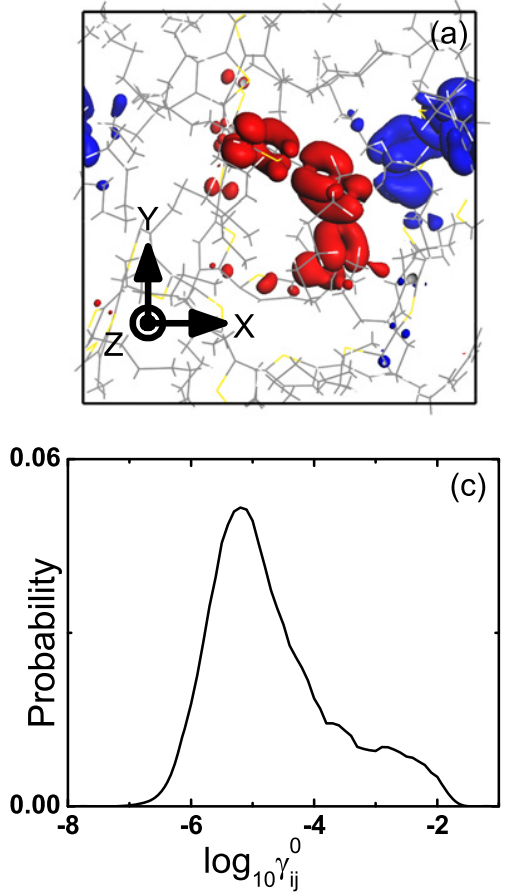

Subroutine calc_mu: calculates the mobility by Eq. (12) with the self-consistently determined carrier concentration.

\subsubsection{MUCal input}

The input files include eigenvalue.dat and bandpos.dat which are obtained from the BOMD code; transrate.dat comes from the TDNA code, and input.dat specifies the parameters in the simulation. A typical input.dat is listed below, followed by the meaning of the parameters:

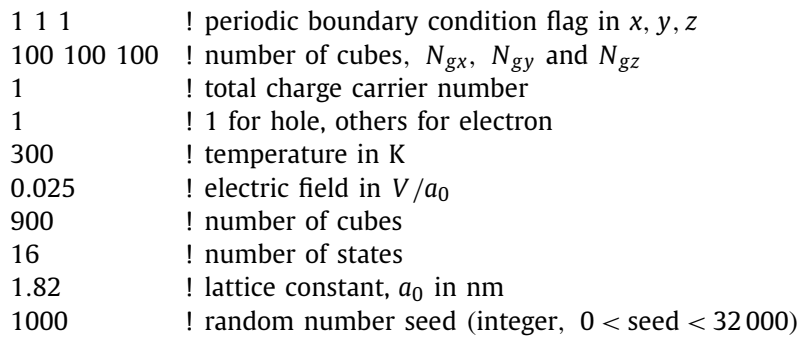

For the periodic boundary condition flag in the first line, 1 stands for the periodic boundary condition and 0 for the open boundary condition (vacuum) in that direction. For the random number seed, a different value indicates a different choice of random cube selection and thus a different macroscopic configuration.

\subsubsection{MUCal output}

The output of this code is output.dat. In this file, some intermediate results are provided, and the final result of the mobility is given at the last line.

\section{Hole mobility of P3HT polymer}

As an example, the hole mobility of an amorphous P3HT polymer system is calculated with the program. The detailed input
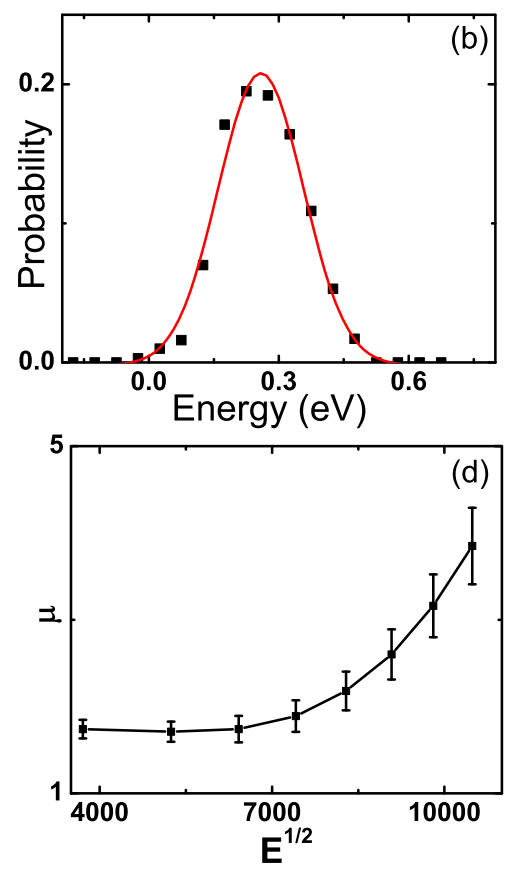

Fig. 4. The hole mobility of the amorphous P3HT polymer. (a) The initial structure of the disordered P3HT and the wave-function isosurface $\left(0.01 e / \AA^{3}\right)$ of two typical states. (b) The distribution of the HOMO energy (solid square) during 1000 step micro-canonical MD simulations and the red curve is the Gaussian fit. (c) The distribution of inter-state transition rates (unit in $\mathrm{fs}^{-1}$ ). (d) The mobility (in $10^{-9} \mathrm{~m}^{2} / \mathrm{V} \mathrm{s}$ ) vs. electric field (in $\mathrm{V} / \mathrm{m}$ ) at $300 \mathrm{~K}$ with the carrier concentration of $10^{-6} e /$ cube averaged over 10 different macroscopic configurations; the error bar shows the standard deviation of the mobility. (For interpretation of the references to color in this figure legend, the reader is referred to the web version of this article.) 
and output files can be found in the example files. The macroscopic system consists of $100 \times 100 \times 100$ cubes and each cube has a dimension of $1.8 \mathrm{~nm}$. Each cube contains 606 atoms, including three P3HT chains with each chain of eight thiophene rings, which leads to a mass density of $1.1 \mathrm{~g} / \mathrm{cm}^{3}$, the same as the experimental value [16]. The top 16 valence bands are chosen as the relevant states of the hole carrier. The BOMD calculations are carried out for the home cube with the periodic boundary conditions using the VASP package [10]. The initial structure of the P3HT chains starting from a randomly placed and warped configuration is fully relaxed to reach the local energy minimum. The $a b$ initio BOMD simulations are performed to bring the system to $300 \mathrm{~K}$ with repeated velocity scaling. The system is then kept at the desired temperature for $500 \mathrm{fs}$ with 1 fs time-step to reach the thermal equilibrium. Subsequently, the micro-canonical production run is carried out for 1000 fs with 1 fs time-step based on a slightly modified VASP code. The eigenvalues, coupling matrix elements and wave-function positions are generated in the output files. Finally, the MuCal code is run to determine the hole mobility for the macroscopic system as a function of the uniformly applied electronic fields at the carrier density of $10^{-6} e$ /cube.

Fig. 4(a) shows two representative hole states in the initial structure, with the coordinates $r=(16.1,13.0,4.9)$ for the blue state and $r=(9.9,9.2,10.0)$ for the red state (unit in $\AA$ ). The localization of the states is evident. From the results of the BOMD code, we calculate the energy distribution of the highest-occupiedmolecular-orbital (HOMO) level as shown in Fig. 4(b). This distribution is fitted by a Gaussian function with a width $\sigma=0.10 \mathrm{eV}$. This width is very close to the experimental value of $0.098 \mathrm{eV}$ [17]. In Fig. 4(c), we show the distribution of the transition rates calculated from the TDNA code. The inter-state transition rates range from $10^{-6}$ to $10^{-2} \mathrm{fs}^{-1}$ with the main peak around $10^{-5} \mathrm{fs}^{-1}$. The mobility vs. electric field curves are shown in Fig. 4(d). The mobility at low fields is about $2 \times 10^{-9}$, which is consistent with the experimental measurement of $2.8 \times 10^{-9}$ [18]. The more detailed results and discussions can be found elsewhere [7].

\section{Summary}

In summary, we have developed a first-principles based Fortran program to calculate the carrier mobility in disordered semiconductors. The first-principles BOMD and non-adiabatic MD are performed to determine the microscopic and macroscopic transition rates. The master equation is solved self-consistently to calculate the carrier mobility as a function of applied electric field, temperature and carrier concentration. There is no empirical input or adjustable parameter in the program, which makes it a general and reliable computational tool for calculating carrier mobility in disordered semiconductors. The program can be very valuable for rational design of organic electronic materials.

\section{Acknowledgement}

The work was supported by NSF Solar grant DMR-1035480.

\section{References}

[1] J.H. Burroughes, D.D.C. Bradley, A.R. Brown, R.N. Marks, K. Mackay, R.H. Friend, P.L. Burns, A.B. Holmes, Light-emitting-diodes based on conjugated polymers, Nature 347 (6293) (1990) 539-541.

[2] H. Sirringhaus, P.J. Brown, R.H. Friend, M.M. Nielsen, K. Bechgaard, B.M.W. Langeveld-Voss, A.J.H. Spiering, R.A.J. Janssen, E.W. Meijer, P. Herwig, D.M. de Leeuw, Two-dimensional charge transport in self-organized, high-mobility conjugated polymers, Nature 401 (6754) (1999) 685-688.

[3] N.S. Sariciftci, D. Braun, C. Zhang, V.I. Srdanov, A.J. Heeger, G. Stucky, F. Wudl, Semiconducting polymer-buckminsterfullerene heterojunctions - diodes, photodiodes, and photovoltaic cells, Appl. Phys. Lett. 62 (6) (1993) 585-587.

[4] V. Coropceanu, J. Cornil, D.A. da Silva, Y. Olivier, R. Silbey, J.L. Bredas, Charge transport in organic semiconductors, Chem. Rev. 107 (4) (2007) 926-952.

[5] W.R. Duncan, W.M. Stier, O.V. Prezhdo, Ab initio nonadiabatic molecular dynamics of the ultrafast electron injection across the alizarin- $\mathrm{TiO}_{2}$ interface, J. Am. Chem. Soc. 127 (21) (2005) 7941-7951.

[6] Z.G. Yu, D.L. Smith, A. Saxena, R.L. Martin, A.R. Bishop, Molecular geometry fluctuations and field-dependent mobility in conjugated polymers, Phys. Rev. B 63 (8) (2001) 085202.

[7] X. Zhang, Z. Li, G. Lu, First-principles determination of charge carrier mobility in disordered semiconducting polymers, Phys. Rev. B 82 (20) (2010) 205210.

[8] H. Bassler, Charge transport in disordered organic photoconductors - a MonteCarlo simulation study, Phys. Status Solidi B 175 (1) (1993) 15-56.

[9] P.V. Parandekar, J.C. Tully, Mixed quantum-classical equilibrium, J. Chem. Phys. 122 (9) (2005) 094102.

[10] G. Kresse, J. Furthmuller, Efficient iterative schemes for ab initio total-energy calculations using a plane-wave basis set, Phys. Rev. B 54 (16) (1996) 1116911186.

[11] X. Gonze, G.M. Rignanese, M. Verstraete, J.M. Beuken, Y. Pouillon, R. Caracas, F. Jollet, M. Torrent, G. Zerah, M. Mikami, P. Ghosez, M. Veithen, J.Y. Raty, V. Olevano, F. Bruneval, L. Reining, R. Godby, G. Onida, D.R. Hamann, D.C. Allan, A brief introduction to the abinit software package, Z. Kristallogr. 220 (5-6) (2005) 558-562.

[12] P. Giannozzi, S. Baroni, N. Bonini, M. Calandra, R. Car, C. Cavazzoni, D. Ceresoli, G.L. Chiarotti, M. Cococcioni, I. Dabo, A. Dal Corso, S. de Gironcoli, S. Fabris, G. Fratesi, R. Gebauer, U. Gerstmann, C. Gougoussis, A. Kokalj, M. Lazzeri, L. Martin-Samos, N. Marzari, F. Mauri, R. Mazzarello, S. Paolini, A. Pasquarello, L. Paulatto, C. Sbraccia, S. Scandolo, G. Sclauzero, A.P. Seitsonen, A. Smogunov, P. Umari, R.M. Wentzcovitch, Quantum espresso: a modular and open-source software project for quantum simulations of materials, J. Phys. Condens. Matter 21 (39) (2009) 395502.

[13] J.M. Soler, E. Artacho, J.D. Gale, A. Garcia, J. Junquera, P. Ordejon, D. SanchezPortal, The siesta method for ab initio order-n materials simulation, J. Phys. Condens. Matter 14 (11) (2002) 2745-2779.

[14] http://www.cpmd.org.

[15] http://hpcrd.lbl.gov/ linwang/petot/petot.html.

[16] J. Mardalen, E.J. Samuelsen, O.R. Gautun, P.H. Carlsen, Chain configuration of poly(3-hexylthiophene) as revealed by detailed X-ray-diffraction studies, Solid State Commun. 77 (5) (1991) 337-339.

[17] C. Tanase, E.J. Meijer, P.W.M. Blom, D.M. de Leeuw, Unification of the hole transport in polymeric field-effect transistors and light-emitting diodes, Phys. Rev. Lett. 91 (21) (2003) 216601.

[18] N.I. Craciun, J. Wildeman, P.W.M. Blom, Universal Arrhenius temperature activated charge transport in diodes from disordered organic semiconductors, Phys. Rev. Lett. 100 (5) (2008) 056601. 